\title{
Performance Evaluation of DVMRP Multicasting Network over ICMP Ping Flood for DDoS
}

\author{
Ashish Kumar \\ Dr. B R Ambedkar National \\ Institute of Technology, \\ Jalandhar
}

\author{
Ajay K Sharma \\ Dr. B R Ambedkar National \\ Institute of Technology, \\ Jalandhar
}

\author{
Arun Singh \\ Dr. B R Ambedkar National \\ Institute of Technology, \\ Jalandhar
}

\begin{abstract}
The Paper evaluates the performance of Distance Vector Multicast Routing Protocol (DVMRP) Multicasting Network with two sources and four receivers, attack by five attackers. Attackers attacked the source 1 in network by ICMP Ping Flood. Here we execute the simulation and draw network throughput between source 1 and source 2, queuing transmission delay and drop out data packets at source 1 for ping packet of size 16, 64 and 96 Bytes for 500, 1500 and 2500 ping packets per second. The simulation results indicate that throughput decreases with the increase in attack packet size and intensity, delay increases with increase in attack packet size and decrease with increase in attacking intensity. Also, with the increase in attack packet size the number of dropped packets remain nearly same for different intensities.
\end{abstract}

\section{Keywords}

DVMRP, Drop Tail, DDoS, PING Flood, Data Multicasting, NS2

\section{INTRODUCTION}

Multicasting is a widely used service in today's computer networking system; it is mostly used in Streaming media, Internet television, video conferencing and net meeting etc. Routers involved in multicasting packets need a better management over stacking system of packets to be multicast. Quality of service (QOS) is also dependent on the availability of the system. It is a very critical issue for the growth of the society.

The "availability" of services means that the information, the systems and the security controls are all accessible and operable in committed state at some random point of time [1].However, the inherent vulnerabilities [2] of the internet architecture provides opportunities for a lot of attacks on the services. Distributed denial-of-service (DDoS) attack is one of such kind which is a threat to the availability of the services. It reduces or completely disrupts services to legitimate users by expending communication and or computational resources of the target [2]. DDoS attacks are amplified form of DoS attacks where attackers direct hundreds or thousands of compromised hosts called zombies against single target $[3,4]$.

Ping flooding [5] is one of the kinds of DDoS attacks. ICMP ECHO_REQUEST message is send to host system to check the connectivity and it expects ECHO_REPLY. In ping flooding many systems is used to send multiple requests to the target system. Ping Flood attacks attempt to saturate a network by sending a continuous series of ICMP echo requests (pings) over a high bandwidth connection to a target host on a lower-bandwidth connection to cause it to send back an ICMP echo reply for each request. Ping Flood attacks can slow down a network or even disable network connectivity [6].

A Distance Vector Multicast Routing Protocol is a dense mode protocol. It is the first protocol used for multicasting. DVMRP uses reverse path forwarding to forward the data over the network. So when a router receives a packet it floods the packet out of all paths to reach all LANs except the one that leads back to the source of the packet. The queuing algorithm used in simulation is Drop Tail. Drop Tail object, which implements First in First out (FIFO) scheduling and drop-on-overflow buffer management typical of most present day Internet routers [7].

The rest of the paper is organized as follow. Section II describes the system topology, multicasting, DVMRP, Queuing method, DDoS attack and Attack scenario. In section III simulation results are discussed without attack traffic and with attack traffic. Finally, we conclude our paper in section IV.

\section{SYSTEM DESCRIPTION}

\subsection{Topology}

A network of six nodes is created and UDP protocol is used to send constant bit rate (CBR) packets. Bandwidth is $0.5 \mathrm{Mbps}$ between node $(2-4)$, node $(4-5)$, node $(4-6)$ and node $(5$ $6)$, and all other connections have a bandwidth of $0.3 \mathrm{Mbps}$, delay of $10 \mathrm{~ms}$; node 1 and node 2 is the data source and multicast protocol will be put into effect at $0.4 \mathrm{~s}$ and $2 \mathrm{~s}$ respectively in the two node; receiver nodes $3,4,5$ and 6 will be effective at $0.6 \mathrm{~s}, 1.3 \mathrm{~s}, 1.6 \mathrm{~s}$, and $2.3 \mathrm{~s}$ respectively; node 4 and node 3 will leave the group at $1.9 \mathrm{~s}$ and $3.5 \mathrm{~s}$.

The node 1 and node 2 is the source node which refers to node 0 and node 1 in the topology and can be seen from the topology is as fig 1.Other nodes are marked as receivers, the topology is coded in ns 2 TCL as,

\footnotetext{
\# Topology Layout

\$ns duplex-link \$n(1) \$n(2) 0.3Mb 10ms DropTail

\$ns duplex-link \$n(2) \$n(3) 0.3Mb 10ms DropTail

\$ns duplex-link \$n(2) \$n(4) 0.5Mb 10ms DropTail

\$ns duplex-link \$n(2) \$n(5) 0.3Mb 10ms DropTail

\$ns duplex-link \$n(3) \$n(4) 0.3Mb 10ms DropTail

\$ns duplex-link \$n(4) \$n(5) 0.5Mb 10ms DropTail

\$ns duplex-link \$n(4) \$n(6) 0.5Mb 10ms DropTail
} 
\$ns duplex-link \$n(5) \$n(6) 0.5Mb 10ms DropTail \#Group Activity

\$ns at 0.6 "\$n(3) join-group \$rcvr \$group"

\$ns at 1.3 "\$n(4) join-group \$rcvr \$group"

\$ns at 1.6 "\$n(5) join-group \$rcvr \$group"

\$ns at 1.9 "\$n(4) leave-group \$rcvr \$group"

\$ns at 2.3 "\$n(6) join-group \$rcvr \$group"

\$ns at 3.5 "\$n(3) leave-group \$rcvr \$group"

\#Attackers link

\$ns duplex-link \$n(4) \$n(7) 0.5Mb 10ms DropTail

\$ns duplex-link \$n(2) \$n(7) 2Mb 10ms DropTail

\$ns duplex-link \$n(7) \$n(12) 0.5Mb 10ms DropTail

\$ns duplex-link \$n(7) \$n(8) 0.5Mb 10ms DropTail

\$ns duplex-link \$n(7) \$n(9) 0.5Mb 10ms DropTail

\$ns duplex-link \$n(7) \$n(10) 0.5Mb 10ms DropTail

\$ns duplex-link \$n(7) \$n(11) 0.5Mb 10ms DropTail

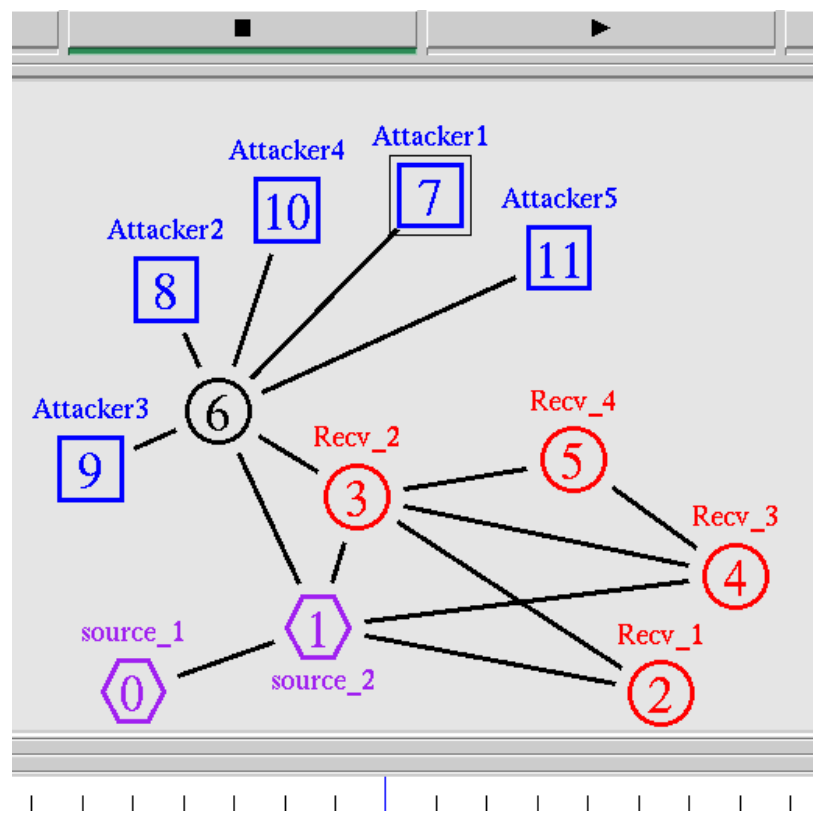

Figure 1 Network Topology Design

\subsection{Multicasting [8]}

The basic principle of multicast routing is that routers must exchange information about neighbouring routers. In order to distribute the data in the network, the designated routers need to establish distribution trees and connect all of the members of a multicast group by accepting the graft message. The distribution trees specify the forwarding path from the source to each of the members of the group. There are a number of different distribution trees, but the two most basic types are source specific trees and shared or centre specific trees.

In source specific trees, the shortest path from the source to the receivers is calculated from the sub networks that are directly connected to the source. Source specific trees build multiple delivery trees to multicast the data.

Shared or centre specific trees use distribution centres and build a single tree that is shared by all members of a group. In the shared tree approach, multicast traffic is sent and received over the same path regardless of the sources of the data.

\section{Multicast routing protocols}

Multicast routing protocols simplify the exchange of information between routers and are responsible for constructing distribution trees and forwarding multicast packets. There are a number of different routing protocols. There are two basic approaches, dense mode or sparse mode.

Dense mode protocols

In Dense mode multicast group members are densely distributed across a network. Because of this, these protocols periodically flood the network with multicast traffic to establish and maintain the distribution tree. Dense mode protocols are suitable for situation where there are a number of hosts that want to or must receive the multicast data and the bandwidth to cope with the flooding of the network.

Sparse mode protocols

The sparse mode multicast group members are sparsely distributed across a network. Group members wanting to receive multicast data are sparsely distributed across a network and that bandwidth is not necessarily widely available. Because the group members are spread sparsely throughout the network, flooding would waste bandwidth and could cause performance problems. They start with empty distribution trees and only add branches when they receive join requests.

\subsection{DVMRP}

DVMRP is a dense mode multicast routing protocol. It uses unicast routing information base to flood multicast datagrams to all multicast routers connected in the network. It has its own unicast routing protocol, based on hop counts, that determines which interface leads back to the source of the data to separate the paths of multicast and unicast data. It is described in RFC 1075, and widely adopted in multicast network. It constructs source- based multicast trees using the Reverse-Path Multicast (RPM) algorithm. A DVMRP route re- lies on the receiving of "poison reverse"' to maintain a list of dependent routers and determine leaf routers. It uses prune messages to prevent future messages from propagating to routers without group membership information.

It assumes that when a source starts sending, members in the network want to receive multicast datagrams. To enable DVMRP mode in NS2 TCL as,

\#Selecting Multicast protocol

DM set CacheMissMode dvmrp

set mproto DM

\#Allocate Group Address

set group [Node allocaddr]

\#All Nodes will contain multicast protocol agents

set mrthandle [\$ns mrtproto \$mproto] 
The prune message has a life time set with it. Once the lifetime expires, multicast datagram will be forwarded again to the previously removed/pruned branches.

Graft messages are used when a new member for a group appears in a pruned area. The router sends a graft message toward the source for the group to turn the pruned branch back into a forwarding branch for multicast messages.

\subsection{Queuing Method}

Drop Tail is a Passive Queue Management (PQM) algorithm which only sets a maximum length for each queue at router [9]. Routers decide when to drop packets. It uses first in first out algorithm. In Drop Tail, the traffic is not differentiated. Each packet is has the same priority. When the queue buffer is filled to its maximum capacity, the packets arrived afterward are dropped till the queue is full. That is, Drop Tail will keep discarding/dropping the packet until the queue has enough room for new packets.

\subsection{DDoS Attack}

Distributed denial of service (DDoS) [10] is a major threat to the availability of Internet services. The goal of a DDoS attack is to completely tie up the resources of a server, which prevents legitimate users from accessing the service [11] or providing legitimate service.

There are several types of attacks are there in DDoS, some of these [12]

\subsubsection{SYN Attack:}

A SYN flood attack occurs when a network becomes so overwhelmed by SYN packets initiating incomplete able connection request that it can no longer process legitimate connection requests, resulting in a denial of service (DoS).

\subsubsection{ICMP Flood:}

An ICMP flood occurs when ICMP pings overload a system with so many echo requests that the system expends all its resources responding until it can no longer process valid network traffic. These packets request reply from the victim and this has as a result the saturation of the bandwidth of the victim's network connection [13, 14]. When enabling the ICMP flood protection feature, administrators can set a threshold that once exceeded invokes the ICMP flood attack protection

feature.

$$
\text { host a }
$$

PING

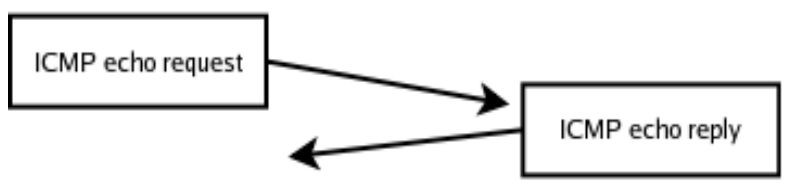

Figure 2 Ping Request flow

\subsubsection{UDP Flood:}

Similar to the ICMP flood, UDP flooding occurs when UDP packets are sent with the purpose of slowing down the system to the point that it can no longer handle valid connections. After enabling the UDP flood protection feature, administrators can set a threshold that once exceeded invokes the UDP flood attack protection feature. (The default threshold value is 1000 packets per second.)

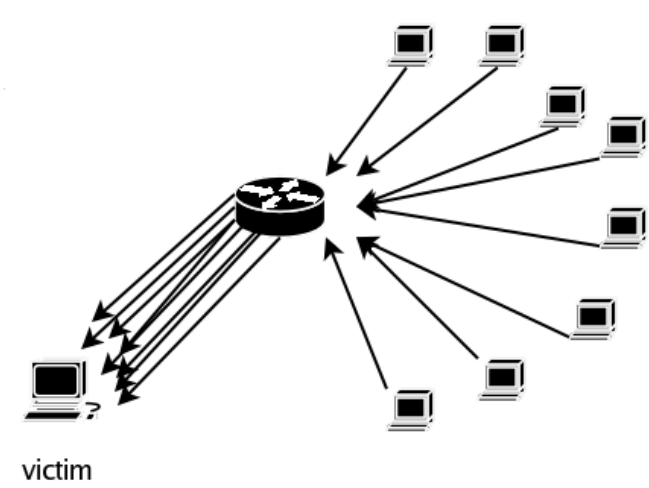

Figure 3 DDoS Attack

Figure 2 show the ping request flow in the network. ICMP echo request is sent by host a and ICMP echo reply is send by host b. Figure 3 depicts an attack situation where multiple systems are compromised to send attack traffic to a target host. In our work ICMP ping packets are used as attack traffic.

\subsection{Attack Scenario for evaluation Purpose}

We have used three different sizes of packet and different intensity i.e. rate of sending ping packets. The table 1 shows the varying packet size with number of ping packets.

Table 1 Intensity and size of ping packets

\begin{tabular}{|c|c|c|c|}
\hline \multirow{2}{*}{$\begin{array}{c}\text { Packet Size } \\
\text { (Bytes) }\end{array}$} & \multicolumn{3}{|c|}{ Intensity (Packet/Sec) } \\
\cline { 2 - 4 } & Scenario 1 & Scenario 2 & Scenario 3 \\
\hline 16 & 500 & 1500 & 2500 \\
\hline 64 & 500 & 1500 & 2500 \\
\hline 96 & 500 & 1500 & 2500 \\
\hline
\end{tabular}

\section{SIMULATION RESULT}

\subsection{Without Attack Traffic}

Figure 4 shows the throughput graph of data packets when there is no attack on the network. The throughput gained is $0.3 \mathrm{Mb} / \mathrm{s}$ which is the allocated rate of source 1 . 


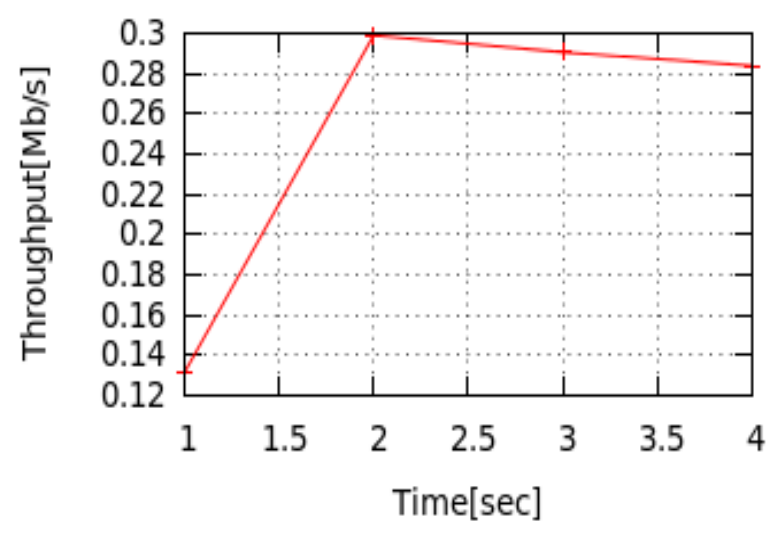

Figure 4 Throughput for source 1

Figure 5 shows the queuing transmission delay graph of packets sent by source 1 and received at node 2 i.e. source 2.The maximum delay is $0.1 \mathrm{~s}$ and average delay is $0.0345364 \mathrm{~s}$.

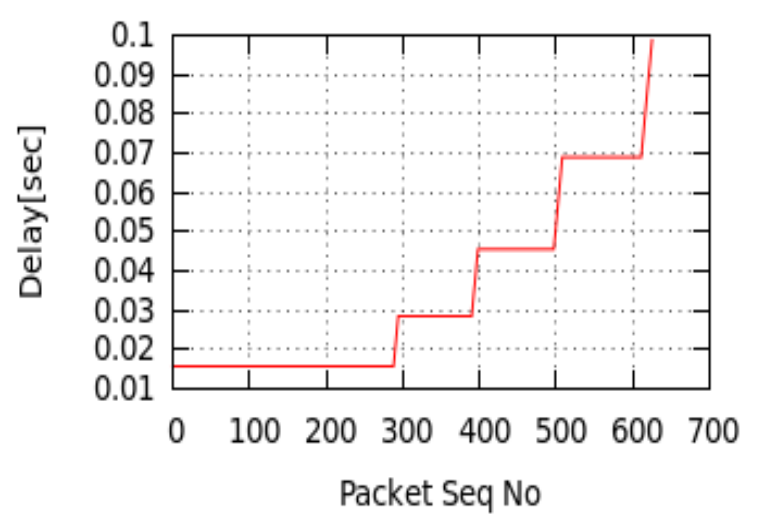

Figure 5 Delay between source 1 and source 2

In case of no attack traffic there is no packet drop at source 1 since the complete bandwidth is used to send the legitimate data.

\subsection{With attack traffic}

\subsubsection{Throughput}

During a DDoS attack, attacking traffic fills the bottleneck link to drop most of the legitimate packets. In this explanation, we concentrate on the attack period which is started at $0.6 \mathrm{~s}$ and stoped at $3.9 \mathrm{~s}$.

Figure 6 shows the throughput for source 1 at attack packets size 16 byte, when source 1 is attacked by 500, 1500 and 2500 ping packets. The graph shows increase in throughput with decrease in number of attack packets at source 1. Maximum throughput achieved is $0.24696 \mathrm{Mb} / \mathrm{s}$ for attack intensity of $500 \mathrm{pkts} / \mathrm{sec}$, whereas throughput is $0.16632 \mathrm{Mb} / \mathrm{s}$ and $0.10416 \mathrm{Mb} / \mathrm{s}$ for intensity of $1500 \mathrm{pkts} / \mathrm{sec}$ and $2500 \mathrm{pkts} / \mathrm{sec}$, respectively. Simulation shows large throughput for less number of attack packets, as time passes this throughput becomes almost constant for 500 and 1500 number of attack packets but decreases drastically for 2500 number of attack packets which is $0.03192 \mathrm{Mb} / \mathrm{s}$.

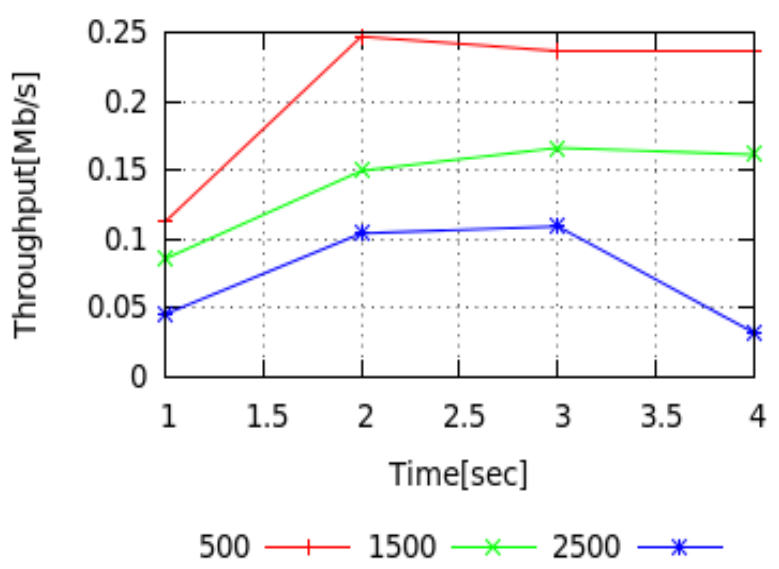

Figure 6 Throughput between source 1 and source2 at 16 bytes packet

Figure 7 shows the throughput for source 1 at attack packets size 64 byte, when source 1 is attacked by 500, 1500 and 2500 ping packets. The graph shows increase in throughput with decrease in number of attack packets at source 1. Throughput at attack intensity $1500 \mathrm{pkts} / \mathrm{sec}$ and $2500 \mathrm{pkts} / \mathrm{sec}$ is same till $2 \mathrm{sec}$ of simulation. Maximum throughput of $0.14616 \mathrm{Mb} / \mathrm{s}$ is achieved with attack intensity of $500 \mathrm{pkts} / \mathrm{sec}$, whereas achieved throughput of $0.12264 \mathrm{Mb} / \mathrm{s}$ and $0.11088 \mathrm{Mb} / \mathrm{s}$ with attack intensity of $1500 \mathrm{pkts} / \mathrm{sec}$ and $2500 \mathrm{pkts} / \mathrm{sec}$ respectively. Simulation shows large throughput for less number of attack packets, as time passes this throughput becomes almost constant for 1500 and 2500 number of attack packets but increases drastically for 500 number of attack packets.

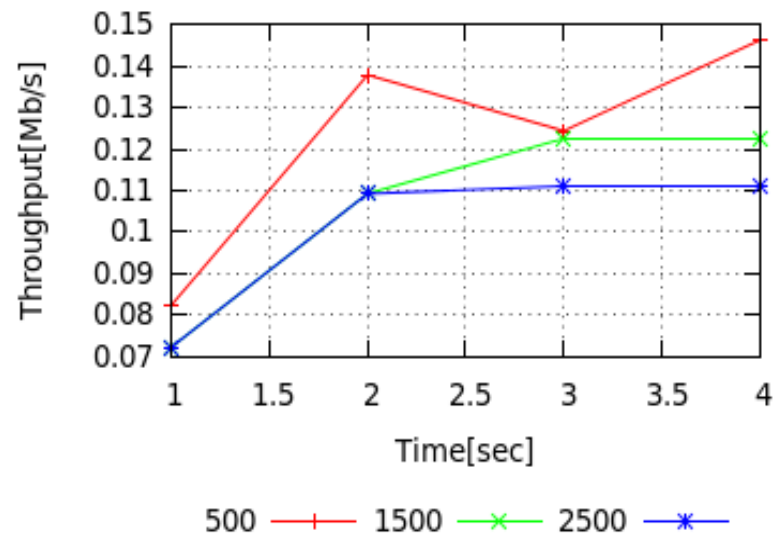

Figure 7 Throughput between source 1 and source 2 at 64bytes packet

Figure 8 shows the throughput for source 1 at attack packets of size 96 byte, when source 1 is attacked by 500,1500 and 2500 ping packets. The graph shows increase in throughput with decrease in number of attack packets at source 1 . Throughput at all attack intensity is same till $2 \mathrm{sec}$ of simulation. Maximum throughput of $0.15288 \mathrm{Mb} / \mathrm{s}$ is achieved with the attack intensity is $500 \mathrm{pkts} / \mathrm{sec}$, whereas at intensity $1500 \mathrm{pkts} / \mathrm{sec}$ and $2500 \mathrm{pkts} / \mathrm{sec}$, throughput is $0.13272 \mathrm{Mb} / \mathrm{s}$ and $0.12936 \mathrm{Mb} / \mathrm{s}$ respectively. Simulation shows large throughput for less number of attack packets, as time passes this throughput becomes almost constant for 500 but decreases for 1500 and 2500 number of attack packets after $3 \mathrm{~s}$ of simulation. 


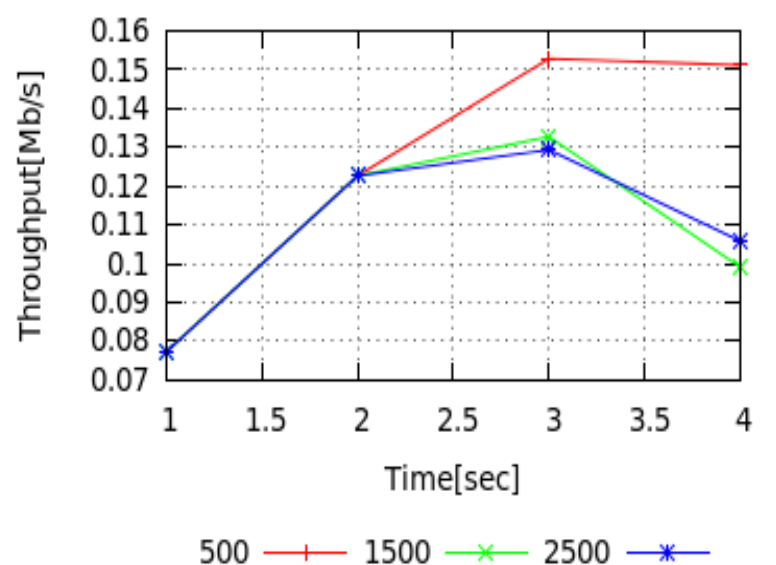

Figure 8 Throughput between source 1 and source 2 at 96 bytes packet

\subsubsection{Queuing Transmission Delay}

Figure 9 shows queuing transmission delay of data packet between source 1 and source 2 for 500, 1500 and 2500 number of attack packets at source 1 for attack packet of size 16 bytes. Average queuing transmission delay at attack intensity $500 \mathrm{pkts} / \mathrm{sec}, 1500 \mathrm{pkts} / \mathrm{sec}$ and $2500 \mathrm{pkts} / \mathrm{sec}$ is $0.0940113,0.0530815$ and 0.0447767 seconds respectively. This delay of transmission is always large for small number of attack packets. The delay of transmission decreases with increase in number of attacks packet and becomes almost same.

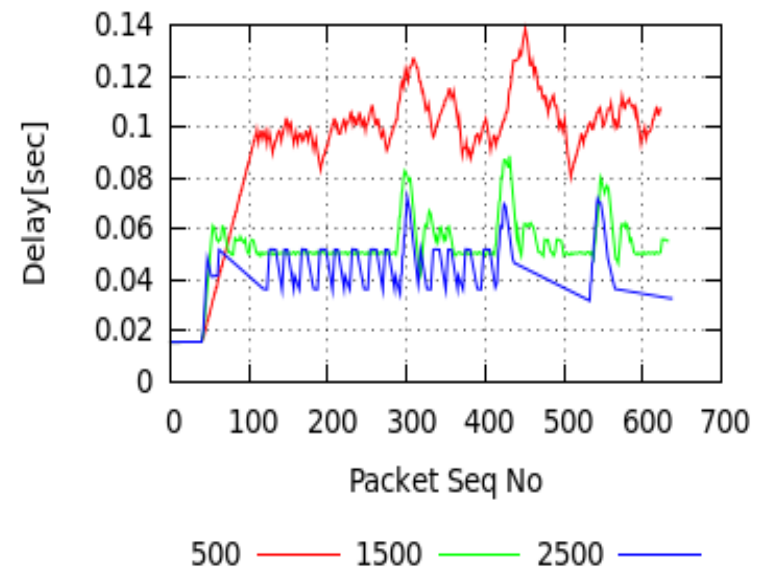

Figure 9 Queuing transmission delay between source 1 and source 2 at 16 bytes attack packets

Figure 10 shows queuing transmission delay of data packet between source 1 and source 2 for 5001500 and 2500 number of attack packets at source 1 for attack packet of size 64 bytes. Average queuing transmission delay at attack intensity $500 \mathrm{pkts} / \mathrm{sec}, 1500 \mathrm{pkts} / \mathrm{sec}$ and $2500 \mathrm{pkts} / \mathrm{sec}$ is 0.128279 , 0.118458 and 0.115442 seconds respectively. This delay of transmission is always large for small number of attack packets. The delay of transmission decreases with increase in number of attacks packet and becomes almost same.

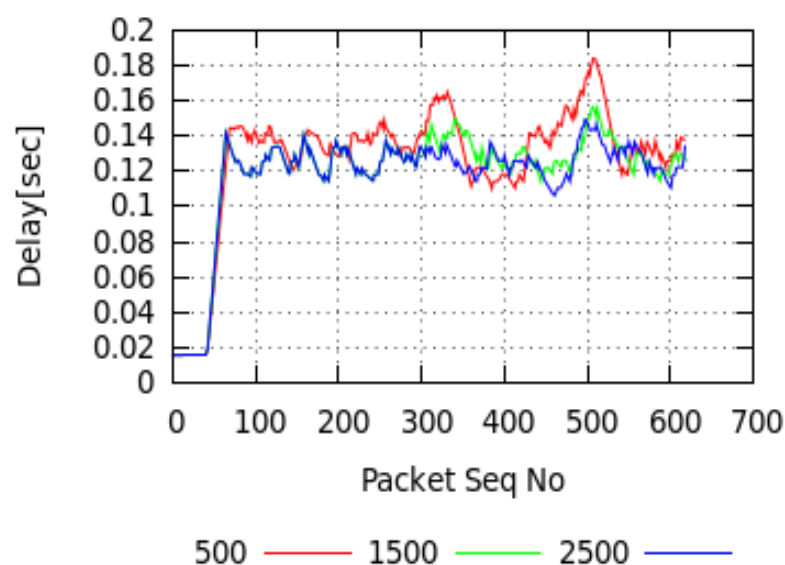

Figure 10 Queuing transmission delay between source 1 and source 2 at 64 bytes attack packets

Figure 11 shows queuing transmission delay of attack packet transmission between source 1 and source 2 for 5001500 and 2500 number of attack packet attack at source 1 for attack packet of size 96 bytes. Average queuing transmission delay at attack intensity 500pkts/sec, $1500 \mathrm{pkts} / \mathrm{sec}$ and $2500 \mathrm{pkts} / \mathrm{sec}$ is $0.165625,0.165625$ and 0.156794 seconds respectively.

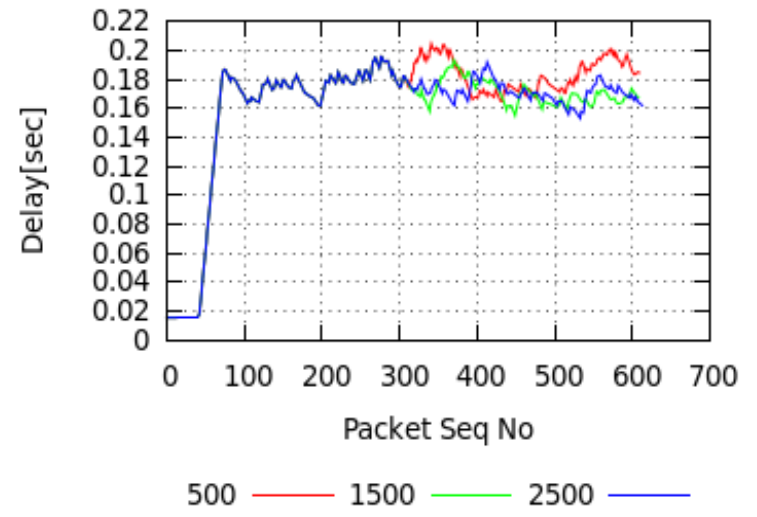

Figure 11 Queuing transmission delay between source 1 and source 2 at 96bytes attack packet

\subsubsection{Drop of Data Packets}

Figure 12 shows the number of drop out packets at source 1 at different attack intensity and attack packet size. In the entire three situations the number of generated packets is 620 . The table 2 shows the number of packets dropped at source 1 in the entire situation.

Table 2 Number of drop out packets at source 1

\begin{tabular}{|c|r|r|r|}
\hline \multirow{2}{*}{$\begin{array}{c}\text { Packet Size } \\
\text { (Bytes) }\end{array}$} & \multicolumn{3}{|c|}{$\begin{array}{c}\text { Intensity } \\
\text { (Packets per second) }\end{array}$} \\
\cline { 2 - 4 } & 500 & 1500 & 2500 \\
\hline 16 & 102 & 271 & 438 \\
\hline 64 & 311 & 354 & 367 \\
\hline 96 & 301 & 344 & 348 \\
\hline
\end{tabular}




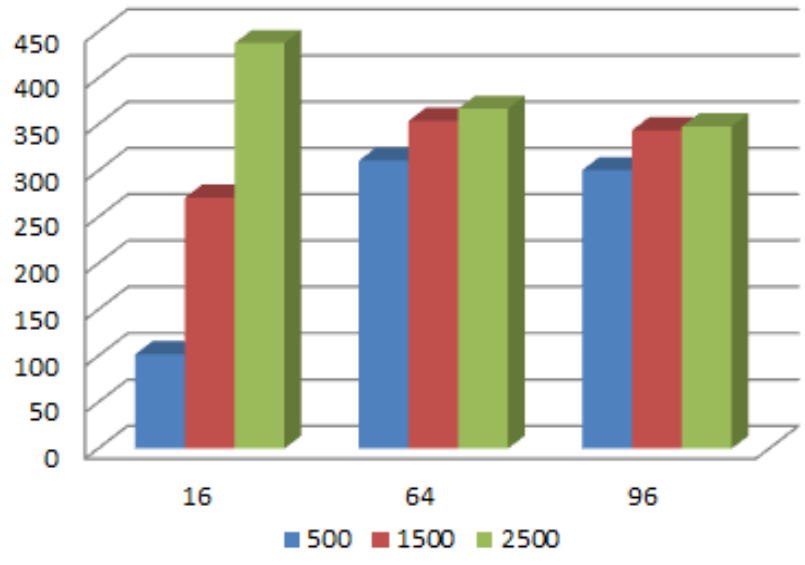

Figure 12 Drop of packets at source 1

\section{CONCLUSIONS}

The results depicts that there is maximum throughput of $0.24696 \mathrm{Mb} / \mathrm{s}$ with attack packet of size 16 Bytes with attacking intensity of 500 ping packets per second. This throughput decreases with increase in attack packet size and attack intensity.

The maximum average transmission delay of $0.165625 \mathrm{~s}$ is calculated for attack packet of size 96 Bytes with attacking intensity of 500 ping packets per second. This delay will increase with increase in attack packet size and decrease with increase in attack intensity.

Drop out data packets are maximum for 16 Bytes at attacking intensity of 2500 ping packets per second. This drop of data packets increases with increase in attack packet size and decreases significantly with attack intensity.

\section{REFERENCES}

[1] Monika Sachdeva, Krishan Kumar, Gurvinder Singh, Kuldip Singh, "Performance Analysis of Web Service under DDoS Attacks", 2009 IEEE International Advance Computing Conference (IACC 2009) Patiala, India, 6-7 March 2009.

[2] J. Mirkovic, D-WARD: Source-End Defense Against Distributed Denial-of-service Attacks, Ph.D. Thesis, University of California, Los Angeles, 2003.

[3] J. Mirkovic and P. Reiher, "A Taxonomy of DDoS Attack and DDoS Defense Mechanisms," ACM SIGCOMM Computer Communications Review, Volume 34, Issue 2, pp. 39-53, April, 2004.
[4] R.K.C. Chang, "Defending against Flooding-Based Distributed Denial-of-Service Attacks: A Tutorial," IEEE Communication Magazine, pp. 42-51, 2002.

[5] Ping Flooding, can be found at: http://tomicki.net/ping.flooding.php

[6] Ping Flood (ICMP Echo) Detection, Can be found at: http://www.daxnetworks.com/Technology/TechDost/T D-101304Ping\%20Flood(ICMP\%20Echo)\%20Detection.pdf

[7] The ns Manual (formerly ns Notes and Documentation), The VINT Project A Collaboration between researchers at UC Berkeley, LBL, USC/ISI, and Xerox PARC. Kevin Fall hkfall@ee.lbl.govi, Editor Kannan Varadhan hkannan@catarina.usc.edui, Editor, May 9, 2010, page no 73 .

[8] Multicasting White paper, Allied Telesis,Can be found at

http://www.alliedtelesis.com/media/pdf/multicasting_w p.pdf

[9] Arash Dana and Ahmad Malekloo,"Performance Comparison between Active and Passive Queue Management" ,IJCSI International Journal of Computer Science Issues, Vol. 7, Issue 3, No5, May 2010

[10] Distributed Denial of Service (DDoS), can be found at http://www.cert.org/homeusers/ddos.html

[11] Shigang Chen, Member,IEEE, and Qingguo Song, (2005), Perimeter- based Defense against Bandwith DDoS Attacks, IEEE Transactions on parallel and Distributed systems, Vol.16,No.6, Digital Object Identifier: 10.1109/TPDS.2005.74.

[12] S.Gavaskar, R.Surendiran, Dr.E.Ramaraj, "Three Counter Defense Mechanism for TCP SYN Flooding Attacks", International Journal of Computer Applications (0975 - 8887) Volume 6- No.6, September 2010.

[13] Christos Douligeris, Aikaterini Mitrokotsa, "DDoS attacks and defense mechanisms: classification and state-of-the-art”, Elsevier, Computer Networks 44 (2004) 643-666.

[14] P.J. Criscuolo, Distributed Denial of Service Trin00, Tribe Flood Network, Tribe Flood Network 2000, and Stacheldraht CIAC-2319, Department of Energy Computer Incident Advisory (CIAC), UCRL-ID136939, Rev. 1, Lawrence Livermore National Laboratory, February 14, 2000, Available from <http://ftp.se.kde.org/pub/security/csir/ciac/ciacdocs/cia c2319.txt>. 